\title{
NEURAL NETWORK PATTERN RECOGNITION \\ OF HANDWRITTEN ARABIC ALPHABET CHARACTERB
}

Fawzy Ibrahim *

\begin{abstract}
The development of handwritten character recognition system has been an old puzzle to the researchers in the field. This is one field where neural networks are expected to achieve new progress. This paper presents the design and implementation of an efficient and reliable recognition software for optically scanned isolated Arabic handwritten characters. The principal goal of the study is to investigate possible applications of neural network models to implement a complete Arabic handwritten document reading system. A neural network architecture based on the backpropagation learning algorithm is used. The system output is a code corresponding to the scanned Arabic letter in a modified version of ASMO character set. This code is chosen because of its compatibility with the most Arabic word processing techniques. The effectiveness of the method has been tested on a data set of 2800 handwritten random samples. Analysis of the obtained results shows that the network accomplishes $98.21 \%$ accuracy.
\end{abstract}

\section{INTRODUCTION}

Machine character recognition has been a major object of pattern recognition for many years. Many handwritten character recognition researches have studied neural network. However, some of these techniques are limited to digits or numerals recognition [1-3], some others are concerned with recognition of English [4], Korean [5], or Japanese [6] characters. Perhaps because of the complexity of Arabic handwritten, as compared to other languages, Arabic character recognition remained almost untouched field until late 1980's [7-9].

Arabic language has an alphabet of 28 basic letters, and 3 other special letters. Each letter has four possible shapes depending on its position within a word, whether it is isolated, initial, medial, or terminal, as shown in the first four columns of Table. 1. The Arabic language has certain characteristics that make it different from English and other languages. These differences will show that Arabic character recognition is not a direct implementation of the recognition techniques used for English. These characteristics include: 1. Arabic text is written

* Lecturer, Department of Electrical and Computer Engineering Military Technical College, Kobry El-Koba, Cairo, Egypt. 
from right to left. 2. Each of the characters can have four different shapes depending on the position in the word. 3. Many of the characters have dots at suitable distance above or below the letter body. Dots can be single, double or triple. 4. Some characters use special marks to modify the letter accent, such as Hamza ( $S$ ), and Madda ( $\sim$ ), again positioned at a certain distance from the letter.

The objective of this work is to recognize various shapes of handwritten Arabic characters which look different almost every time they are written by the same person. Using neural network seemed appropriate to solve this problem.

\section{OPTICAL CHARACTER RECOGNITION SYSTEM}

The general scheme of an optical character recognition is shown in Fig. 1. The input to this system is a scanned or digitized image of a character. The output of this system is the recognized character in one of the following formats: either text or graphics mode, or a code corresponding to an Arabic letter in a modified version of ASMO character set as illustrated by the fifth and sixth columns of Table. 1. This code is used to represent the Arabic characters in the Arabic word processing packages.

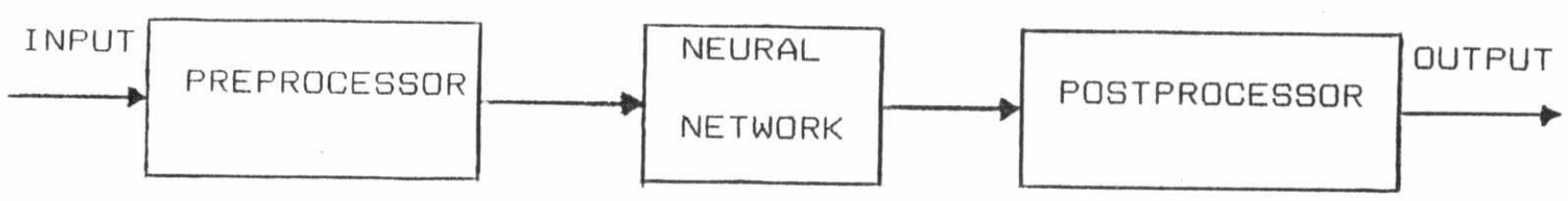

Fig. 1 General scheme for an optical character recognizer.

\subsection{The Preprocessor}

The preprocessing phase is done in the following sequence:

Step 1 An image file of a scanned character is read. The image information is decoded into pixels of 1 's and 0 ' 5 . An example of the second alphabet character " $"$ " is shown in Fig. 2 (a).

Step 2 The image is clipped from the four sides to keep only the useful information in a smaller size, as shown in Fig. 2
(b).

step 3 The dots are identified to determine their numbers and positions, and then removed from the image as shown in Fig. 2 (c). Step 4 The image is compressed to $16 * 16$ and then to $8 * 8$ matrix,
using a compression technique. 


\subsection{The Neural Network Architecture}

A feedforward four-layer network, as shown in Fig. 3 , is used. It can be described as follows:

Input layer: an $8 * 8$ matrix (64 nodes), fully connected to the first hidden layer.

First hidden layer: an $8 * 8$ ( 64 nodes), with a patterned connection scheme to the second, using a $2 * 2$ pattern matrix, and overlap of 1 in both the $x$ and $y$ directions [10].

Second hidden layer: a $7 * 7$ matrix ( 49 nodes), fully connected to the third hidden layer.

Third hidden layer: a $7 * 7$ matrix ( 49 nodes), fully connected to the output layer.

Output layer: an $8 * 1$ matrix ( nodes).

\subsection{Training the Network}

Several samples of every character's shape, ten samples of each shape written by ten different people are prepared in the way explained above (Fig. $2(d)$ ). Then for every input a target code is assigned to form input/target pairs ( see Table. 1). The system is trained using backpropagation learning rule. The training samples are presented to the network, the system parameters (such as the learning rate, the number of cycles...) are adjusted, and its output is tested. This process is repeated until all the trained characters are recognized correctly. The resulting set of weights are saved, and now the system is ready for the retrieval phase.

\subsection{The Postprocessor}

The standard ASCII table does not provide Arabic characters, so the standard ASMO codes have been used for the output. The arabic character shapes are generated and linked to the active ASCII font table.

\section{CONCLUSION}

A model using the feedforward neural network was discussed and proved to be useful for handwritten Arabic character recognition. The system has the ability match a certain pattern to the desired output code. Furthermore, testing the network verified its capability to generalize or to recognize Arabic characters that has never seen before. The system has been tested on a huge number of characters which showed that the recognition rate is $98.21 \%$ as shown in Fig. 4, and the recognition time is equal to 0.15 second on an IBM 386-5X. 
Table. 1 The basic Arabic letters in four different positions and their corresponding ASMO code.

\begin{tabular}{|c|c|c|c|c|c|}
\hline Isolated & Initial & Medial & Terminal & Decimal Code & Binary Code \\
\hline 1 & 1 & L & $L$ & 199 & 11000111 \\
\hline$\varphi$ & ب & ب. & ب & 200 & 11001000 \\
\hline$\ddot{ت}$ & ت & $\ddot{\mu}$ & $\ddot{a}<ت \| \ddot{0}$ & 202 & 11001010 \\
\hline$\therefore$ & $\ddot{~}$ & $\ddot{\theta}$ & ت & 203 & 11001011 \\
\hline ૫. & $ج$ & $ج$ & 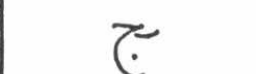 & 204 & 11001100 \\
\hline$\tau$ & $ح$ & $ح$ & $\tau$ & 205 & 11001101 \\
\hline$\dot{\tau}$ & $\dot{~}$ & $\dot{x}$ & $\bar{x}$ & 206 & 11001110 \\
\hline$د$ & $د$ & $\perp$ & 1 & 207 & 11001111 \\
\hline$j$ & j & i & i & 208 & 11010000 \\
\hline ノ. & $\mathcal{J}$ & 5 & 5 & 209 & 11010001 \\
\hline J & J & $j$ & $\dot{s}$ & 210 & 11010010 \\
\hline w & سـ & $-m$ & ver & 211 & 11010011 \\
\hline ش & شُش & ش شت & سُش & 212 & 11010100 \\
\hline ص & $-\varphi$ & $\infty$ & ص & 213 & 11010101 \\
\hline$\dot{\omega}$ & $\dot{\varphi}$ & $\dot{\phi}$ & ن & 214 & 11010110 \\
\hline b & $b$ & b & $b$ & 215 & 11010111 \\
\hline$b$ & b & $\dot{b}$ & b & 216 & $1101 \quad 1000$ \\
\hline$\varepsilon$ & $s$ & s & $\varepsilon$ & 217 & $1101 \quad 1001$ \\
\hline$\varepsilon$ & c & c & $\dot{\xi}$ & 218 & 11011010 \\
\hline ف & $\dot{9}$ & i & $i$ & 225 & 11100001 \\
\hline 9 & $\ddot{g}$ & $\ddot{e}$ & $\ddot{v}$ & 226 & 11100010 \\
\hline (3) & 5 & 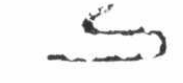 & (5) & 227 & 11100011 \\
\hline$J$ & J & $\curlywedge$ & f & 228 & 11100100 \\
\hline s & $\infty$ & $a$ & $p$ & 229 & 11100101 \\
\hline$\dot{u}$ & ذ & $\dot{~}$ & $\dot{v}$ & 230 & 11100110 \\
\hline D & $D$ & $f$ & 260 & 231 & 11100111 \\
\hline 9 & 9 & 9 & $\vartheta$ & 232 & 11101000 \\
\hline$s$ & $\vec{\sim}$ & $\mu$ & $\checkmark$ & 233 & $1110 \quad 1001$ \\
\hline
\end{tabular}




\section{FIFTH ASAT CONFERENCE}

$\mathrm{CA}-5 \mathrm{513}$

4 - 6 MAY 1993, CAIRO

11111111111111111111111111111111111111111111111111111111111111111111111111111111111111111111111 111111111111111111111111111111111111111111111111111111111111111111111111111111111111111111111111 111111111111111111111111111111111111111111111111111111111111111111111111111111111111111111111111 111111111111111111111111111111111111111111111111111111111111111111111111111111111111111111111111 111111111111111111111111111111111111111111111111111111111111111111111111111111111111111111111111 1111111111111111111111111111111111111111111111111111111111111111111111111111111111111111111111111 1111111111111111111111111111111111111111111111111111111111111111111111111111111111111111111111111 1111111111111111111111111111111111111111111111111111111111111111111111111111111111111111111111111 11111111111111111111111111111111111111111111111111111111111111111111111111111111111111111111111 11111111111111111111111111111111111111111111111111111111111111111111111111111111111111111111111 -1111111111111111111111111111111111111111111111111111111111111111111111111111111111111111111 11111111111111111111111111111111111111111111111111111111111111111111111111111111111111111111111 1111111111111111111111111111111111111111111111111111111111111111111111111111111111111111111111 1111111111111111111111111111111111111111111111111111111111111111111111111111111111111111111

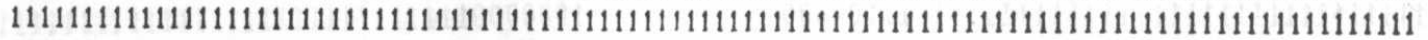
1111111111111111111111111111111111111111111111111111111111111111111111111111111111111111111 1111111111111111111111111111111111111111111111111111111111111111111111111111111111111111111111 11111111111111111111111111000111111111111111111111111111111111111111011111111111111111111111 1111111111111111111111111110000111111111111111111111111111111111111000011111111111111111111 11111111111111111111111111000001111111111111111111111111111111111111100001111111111111111111111 111111111111111111111111111000000111111111111111111111111111111111111000001111111111111111111111 111111111111111111111111111000000111111111111111111111111111111111111000001111111111111111111111 111111111111111111111111110000000111111111111111111111111111111111111000001111111111111111111111 1111111111111111111111111000000011111111111111111111111111111111111101000111111111111111111111 11111111111111111111111110000001111111111111111111111111111111111111000001111111111111111111111 111111111111111111111111111000001111111111111111111111111111111111111000001111111111111111111111 1111111111111111111111111100000111111111111111111111111111111111111000000111111111111111111111 111111111111111111111111111000000111111111111111111111111111111100000000001111111111111111111111 111111111111111111111111111000000011111111111111111111111111000000000000001111111111111111111111 111181111111111111111111111000000000000000001000000000000000000000000000011111111111111111111111 111111111111111111111111111100000000000000000000000000000000000000000001111111111111111111111111 111111111111111111111111111111000000000000000000000000000000000000000011111111111111111111111111 1111111111111111111111111111111100000100000000000000000000000000011111111111111111111111111111111 111111111111111111111111111111111111110000000000000000000000111111111111111111111111111111111111 11111111111111111111111111111111111111111111100111111111111111111111111111111111111111111111111 1111111111111111111111111111111111111111111111111111111111111111111111111111111111111111111111111 11111111111111111111111111111111111111111111111111111111111111111111111111111111111111111111111 111111111111111111111111111111111111111111111111111111111111111111111111111111111111111111111111 i11111111111111111111111111111111111111111111111111111111111111111111111111111111111111111111111 111111111111111111111111111111111111111111111111111111111111111111111111111111111111111111111111 11111111111111111111111111111111111111111111111111111111111111111111111111111111111111111111111 111111111111111111111111111111111111111111111111111111111111111111111111111111111111111111111111 1111111111111111111111111111111111111111111111111111111111111111111111111111111111111111111111111 111111111111111111111111111111111111111111111111111111111111111111111111111111111111111111111 1111111111111111111111111111111111111111111111111111111111111111111111111111111111111111111111111 1111111111111111111111111111111111111111111111111111111111111111111111111111111111111111111111111 111111111111111111111111111111111111111111111111111111111111111111111111111111111111111111111111 11111111111111111111111111111111111111111111111111111111111111111111111111111111111111111111111111 111111111111111111111111111111111111111111111111111111111111111111111111111111111111111111111111 111111111111111111111111111111111111111111111111111111111111111111111111111111111111111111111111 1111111111111111111111111111111111111111111111100011111111111111111111111111111111111111111111 111111111111111111111111111111111111111111111110000011111111111111111111111111111111111111111111 111111111111111111111111111111111111111111111110000011111111111111111111111111111111111111111111 11111111111111111111111111111111111111111111111000001111111111111111111111111111111111111111111 111111111111111111111111111111111111111111111110000011111111111111111111111111111111111111111111 111111111111111111111111111111111111111111111111000011111111111111111111111111111111111111111111 $11111111111111111111111111111111111111111111111000111111 \$ 11111111111111111111111111111111111$ 11111111111111111111111111111111111111111111111111111111111111111111111111111111111111111111 1111111111111111111111111111111111111111111111111111111111111111111111111111111111111111111111 11111111111111111111111111111111111111111111111111111111111111111N11111111111111111111111111111 11111111111111111111111111111111111111111111111111111111111111111111111111111111111111111111111 11111111111111111111111111111111111111111111111111111111111111111111111111111111111111111111111 11111111111111111111111111111111111111111111111111111111111111111111111111111111111111111111111 
110001111111111111111111111111111111111111111101 110000111111111111111111111111111111111111110000 100000111111111111111111111111111111111111110000 100000011111111111111111111111111111111111100000 100000011111111111111111111111111111111111100000 000000011111111111111111111111111111111111100000 000000011111111111111111111111111111111111101000 000000111111111111111111111111111111111111100000 100000111111111111111111111111111111111111100000 100000111111111111111111111111111111111111000000 100000011111111111111111111111111111110000000000 100000001111111111111111111111111100000000000000 100000000000000000100000000000000000000000000001 110000000000000000000000000000000000000000000111 111100000000000000000000000000000000000000001111 111110000010000000000000000000000000001111111111 111111111111000000000000000000000011111111111111 111111111111111111100111111111111111111111111111 1111111111111111111111111111111111111111111111 111111111111111111111111111111111111111111111 11111111111111111111111111111111111111111111111 1111111111111111111111111111111111111111111111 111111111111111111111111111111111111111111111 1111111111111111111111111111111111111111111111 111111111111111111111111111111111111111111111111 11111111111111111111111111111111111111111111111 11111111111111111111111111111111111111111111 11111111111111111111111111111111111111111111111 1111111111111111111111111111111111111111111111 111111111111111111111111111111111111111111111111 1111111111111111111111111111111111111111111 111111111111111111111111111111111111111111111111 11111111111111111111111111111111111111111111 111111111111111111111100011111111111111111111111 111111111111111111111000001111111111111111111111 111111111111111111111000001111111111111111111111 11111111111111111111000001111111111111111111111 1111111111111111111100000111111111111111111111 111111111111111111110000111111111111111111111

110001111111111111111111111111111111111111111101 110000111111111111111111111111111111111111110000 100000111111111111111111111111111111111111110000 10000001111111111111111111111111111111111100000 100000011111111111111111111111111111111111100000 000000011111111111111111111111111111111111100000 000000011111111111111111111111111111111111101000 000000111111111111111111111111111111111111100000 100000111111111111111111111111111111111111100000 100000111111111111111111111111111111111111000000 100000011111111111111111111111111111110000000000 100000001111111111111111111111111100000000000000 100000000000000000100000000000000000000000000001 110000000000000000000000000000000000000000000111 111100000000000000000000000000000000000000001111 111110000010000000000000000000000000001111111111 111111111111000000000000000000000011111111111111

101111111111111 1011111111111110 101111111111110 1001111111111110 1001111111111110 0001111111111110 0001111111111110 001111111111110 1011111111111100 1001111111111000 1001111111110000 1000001000000000 1000000000000001 1100000000000001 1100000000000111 1111000000001111

$$
\text { (c) }
$$
01111110
00111110
00111110
00111110
00111100
ononogno
00000000
10000001

(b)

\footnotetext{
Fig. 2 The image information of the scanned character ( $u)$. (a) the original image. (b) The clipped image.

(c) The dot is removed. (d) the compressed image.
} 


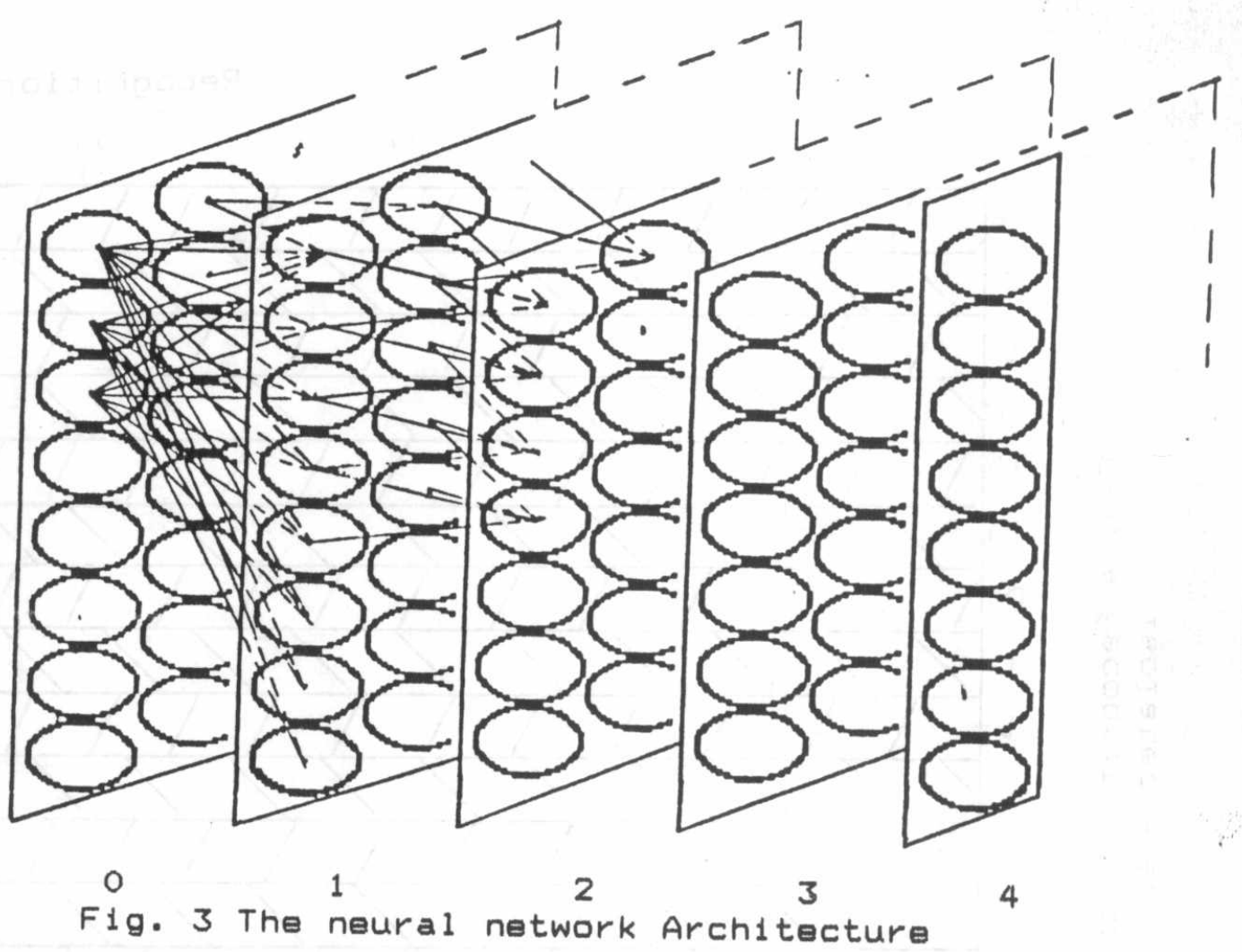

REFERENCES

1. Pawlicki, T. F. and Seihari, S. N., "Neural Network Models and their Applications to Handwritten Digit Recognition, "Proc. IEEE Second Int. Conf. on Neural Network, p. I I-63, San Diego, 1988.

2. Yamada, K. and Temma, T., "Handwritten Numeral Recognition by Multi-layered Neural Network with improved Learning Algorithm, " Proc. IJCNN Int. Joint Conf. on Neural Network, p. II-295,

3. Zhu, $X$. and Umeno, M., "Feature Detector and Application to Handwritten Character recognition, "Proc. IJCNN Int. Joint Conf. on Neural Network, p. I I-457, Wash. D. C. 1990.

4. Burr, D., "Experiments with a Connectionist Text Reader, Proc. IEEE Second Int. Conf. on Neural Network, p. II-7í San Diego, 1988.

5. Lee, K. and Lee, W., "Application of Coulomb Energy Network to Korean Character Recognition, "Proc. IJCNN Int. Joint Conf. on Neural Network, p. I I-635, Wash. D. C. 1990.

6. Haruki, K.and Hatano, H., "Pattern Recognition of Handwritten Phonetic Japanese Alphabet Characters, "Proc. IJCNN Int.

Joint Conf. on Neural Network, p. II-515, Wash. D. C. 1990.

7. Youssef, Y., "An expert Knowledge-based System for Arabic OCR, "Int. Conf. for Stat. and Comp. \& Demo. Res.p-14, Cairo 1989.

8. Goneid, A. and Monir, K., "A Fast Algorithm for the Recogniti ion of Arabic Handwritten Numerals using Topology Features,

9. "Goneid, A. and Hassan AI Applications, P. II-253, Cairo, 1992. Arabic Handwritten Applications, p. II-270, "First Int. Conf. on AI

10. Baffes, P. 1992. Center. U. S. A., 1989. 
Recognition Accuracy [\%]

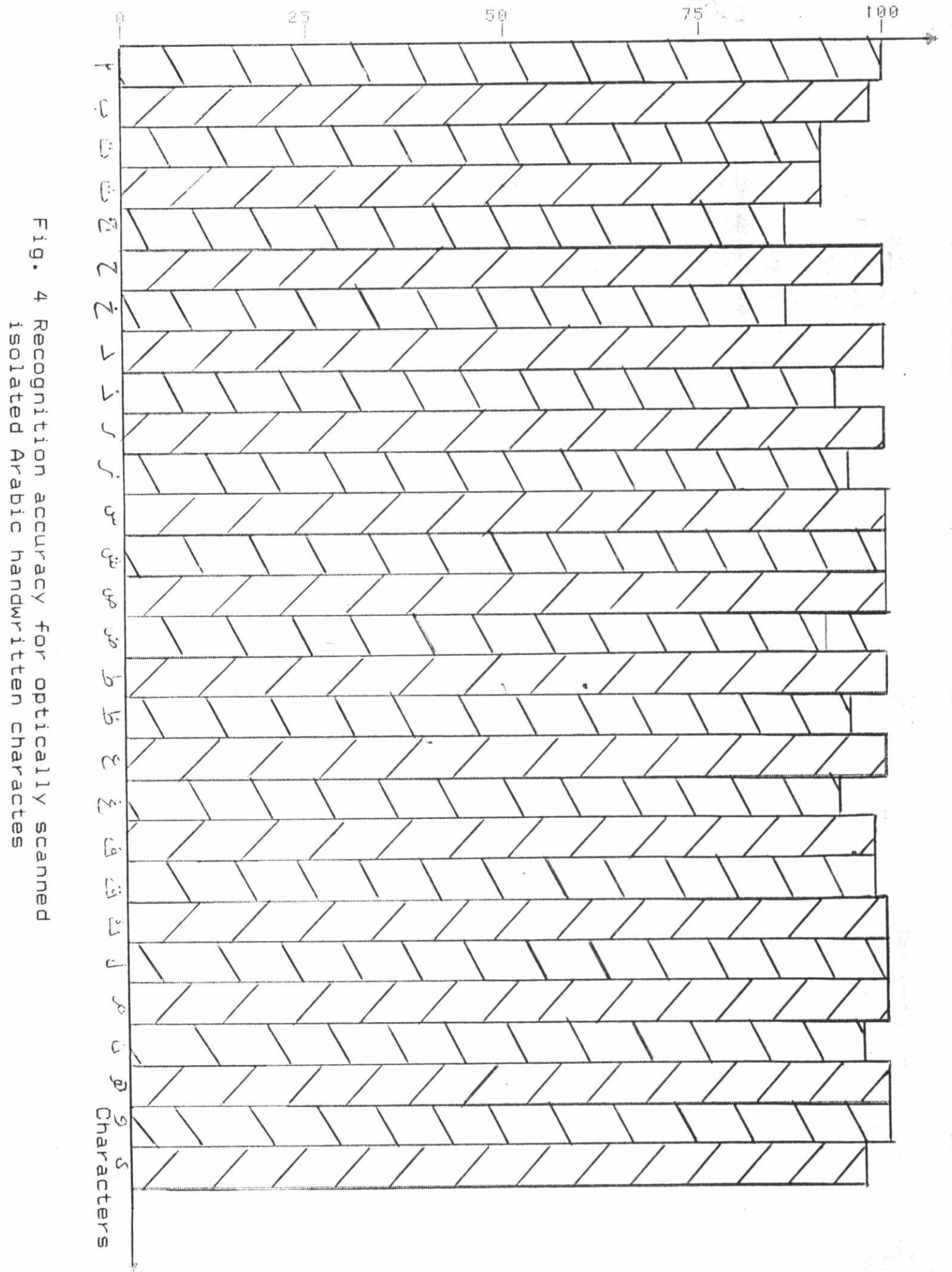

